\title{
IMPACTO DE LAS INVERSIONES EN INFRAESTRUCTURA TURÍSTICA. ARGENTINA
}

\author{
IMPACT OF INVESTMENTS IN TOURISM \\ INFRASTRUCTURE IN ARGENTINA
}

ANA MARÍA GANINO

UNIVERSIDAD NACIONAL DE SAN MARTÍN - UNSAM

ARGENTINA

ADRIÁN GUTIÉRREZ CABELLO

UNIVERSIDAD NACIONAL DE SAN MARTÍN - UNSAM

ARGENTINA

A la república solo ha de salvarla pensar en grande, sacudirse de lo pequeño y proyectar hacia lo porvenir. José Ortega Y Gasset 


\begin{tabular}{c}
\hline ANA MARÍA GANINO - ADRIÁN GUTIÉRREZ CABELLO \\
$>\mathbf{2 8}$ IMPACTO DE LAS INVERSIONES EN INFRAESTRUCTURA TURÍSTICA. ARGENTINA \\
Págs. $27-43$
\end{tabular}

Recibido: 25/08/2016

\title{
RESUMEN
}

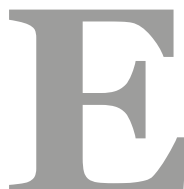

lobjetivo de este trabajo es analizar el impacto de las inversiones en infraestructura en el sector turístico de la Argentina, de acuerdo al plan de inversiones identificado por la Cámara Argentina de la Construcción al año 2014. Este trabajo está elaborado en el marco de un Proyecto de Reconocimiento Institucional promovido por la Universidad Nacional de San Martín - Escuela de Economía y Negocios. El objetivo principal es la creación de un mapa de Inversiones Turísticas en la Argentina, junto con la medición de los impactos socioeconómicos de las mismas.

Palabras Clave: turismo; matriz; insumo; producto; inversiones.

\begin{abstract}
The aim of this article was to analyze the impact of infrastructure investments at the tourism sector of Argentina, according to the investment plan identified by the Argentinan Chamber of Construction in 2014. This study was developed within of a Project Institutional Recognition promoted by the Universidad Nacional de San Martín - Escuela de Economía y Negocios. The main proyect goals were the creaction of a map of tourism investment in Argentina and the analysis of its social and economics impacts.
\end{abstract}

Keywords: tourism; input-output; matrix; investment.

\section{INTRODUCCIÓN}

La realización de las obras de infraestructura destinadas al turismo, de acuerdo a la Cámara Argentina de la Construcción, para el período 2011-2020 están basadas en:

- Construcción y remodelación de hoteles de cuatro y cinco estrellas.

- Centros comerciales.

De acuerdo a las proyecciones realizadas por esta entidad a precios de 2013 significaban una inversión de 29.000 millones de pesos. En la matriz insumo producto ${ }^{1}$ utilizada, para rea- 
lizar la simulación, se expone como incremento en la demanda final de la actividad construcción en infraestructura, en este caso en el componente formación bruto de capital.

El horizonte del desarrollo de este tipo de obras es de alrededor de dos años y medio, por lo tanto el crecimiento esperado en todos los sectores, tanto en el valor de producción, agregado, empleo, etc., a fin de evitar posibles sobreestimaciones se divide por 2,5 los distintos resultados para poder anualizarlos.

En la medición de los impactos directos e indirectos, se analizan los efectos sobre la producción, valor agregado, ocupación, recaudación fiscal e importaciones. Tanto a nivel nacional como provincial, en este caso especialmente como base de partida para el desarrollo del mapa de inversiones.

\section{REGULACIÓN}

Este indicador permite estimar el valor total de los bienes y servicios producidos por la economía independientemente de si tratan de insumos o bienes intermedios utilizados en los procesos productivos o se destinan a un usuario final.

El multiplicar de sobre la producción de la construcción en infraestructura es de 1,9; esto implica que por cada peso que crece esta actividad el resto de la economía lo hace en o,90 pesos. De este modo, estima como impacta la construcción que casi duplica su valor generando un círculo virtuoso.

Los principales sectores beneficiados por este desarrollo de la actividad son:

- Industria cementera y vidrio

- Siderurgia e industria metalmecánica

- Industria maderera y muebles

- Fabricación de pinturas

- Servicios profesionales

- Comercio

\section{IMPACTO SOBRE EL VALOR BRUTO DE PRODUCCIÓN}

En la medición del impacto sobre el valor agregado, el multiplicador que tiene la construcción es de 2, ello significa que ante el incremento en un peso del VAB de este sector en toda la economía se generan dos pesos. Con este indicador, queda expuesta la importancia que tiene esta actividad para la economía y en este caso para el desarrollo de la infraestructura turística. 
Este crecimiento esperado en la construcción significaría el o,17\% del PBI del año 2014. Tal como se mencionó, dada la duración de las obras, los efectos en la expansión de esta actividad se mantienen en cada obra, durante tres años.

Es válido aclarar que a los fines de simplificar la simulación, la ejecución de las obras se realiza al mismo momento y con igual duración. Como cabe esperar en general, los sectores que más explican el crecimiento del VBP, son aquellos que también lo hacen con respecto al VAB.

Tres de cada cuatro pesos se originan en el sector secundario de la economía, de los cuales la industria representa aproximadamente el 27\%, el comercio y los servicios el $21 \%$ y el sector primario representado principalmente por la explotación de minas y canteras el $4 \%$. La importancia de la generación de valor agregado para la economía radica en que una parte de éste es el pago de salarios.

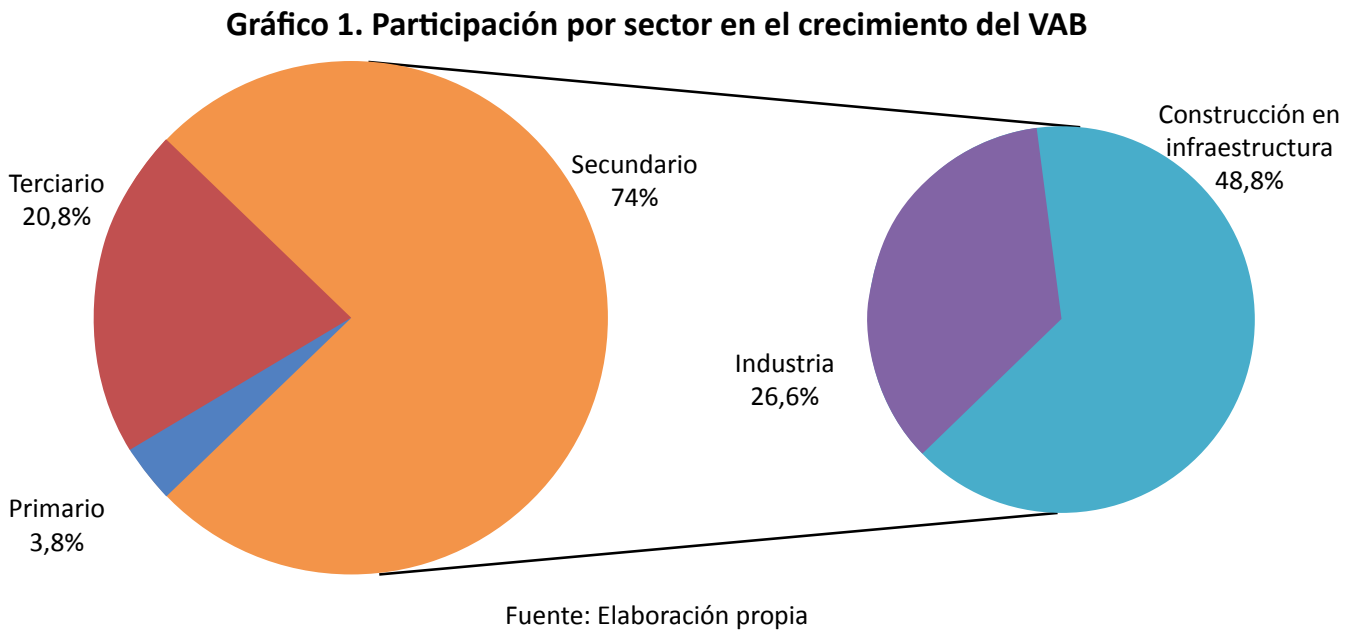

Si bien la mitad del crecimiento del VAB corresponde al sector de la construcción los efectos a nivel provincial se supondrán de acuerdo a la proporcionalidad que tiene cada distrito en el total de las inversiones, de este modo se localizan los impactos directos del VAB por cada provincia.

Pero en la consideración total de los efectos sobre la economía, además de la construcción se consideran los impactos de las demás actividades que son proveedoras de esta y que no necesariamente se corresponde con la localización geográfica de las obras.

Por lo tanto analizando desde las perspectivas de políticas públicas que fomenten el desarrollo sustentable de la actividad económica y en particular la turística se analiza como impacta en cada provincia el desarrollo de estas obras.

Dada la concentración de las obras y la importancia relativa que tiene en la industria y en menor medida en los servicios, el 43,5\% del crecimiento del VAB se origina en la provincia de Buenos Aires, seguido por la Ciudad de Buenos Aires, con el 18,6\%. En estos dos distritos se explican casi en partes iguales los efectos de la construcción y del resto de las actividades. 
En tercer lugar, la beneficiada sería la provincia patagónica de Río Negro, con el 8\%. En este caso, el 92\% de este valor se explica por las obras de infraestructura. Este es un ejemplo de la importancia de estas actividades que permiten el desarrollo provincial y regional, que no se lograría sino es por éstas.

En tanto Santa Fe y Córdoba representan el 6,1\% y 5,1\% respectivamente del crecimiento, en especial por la actividad industrial que se lleva a cabo en estas provincias.

\section{Gráfico 2. Participación por provincia del crecimiento del VAB}

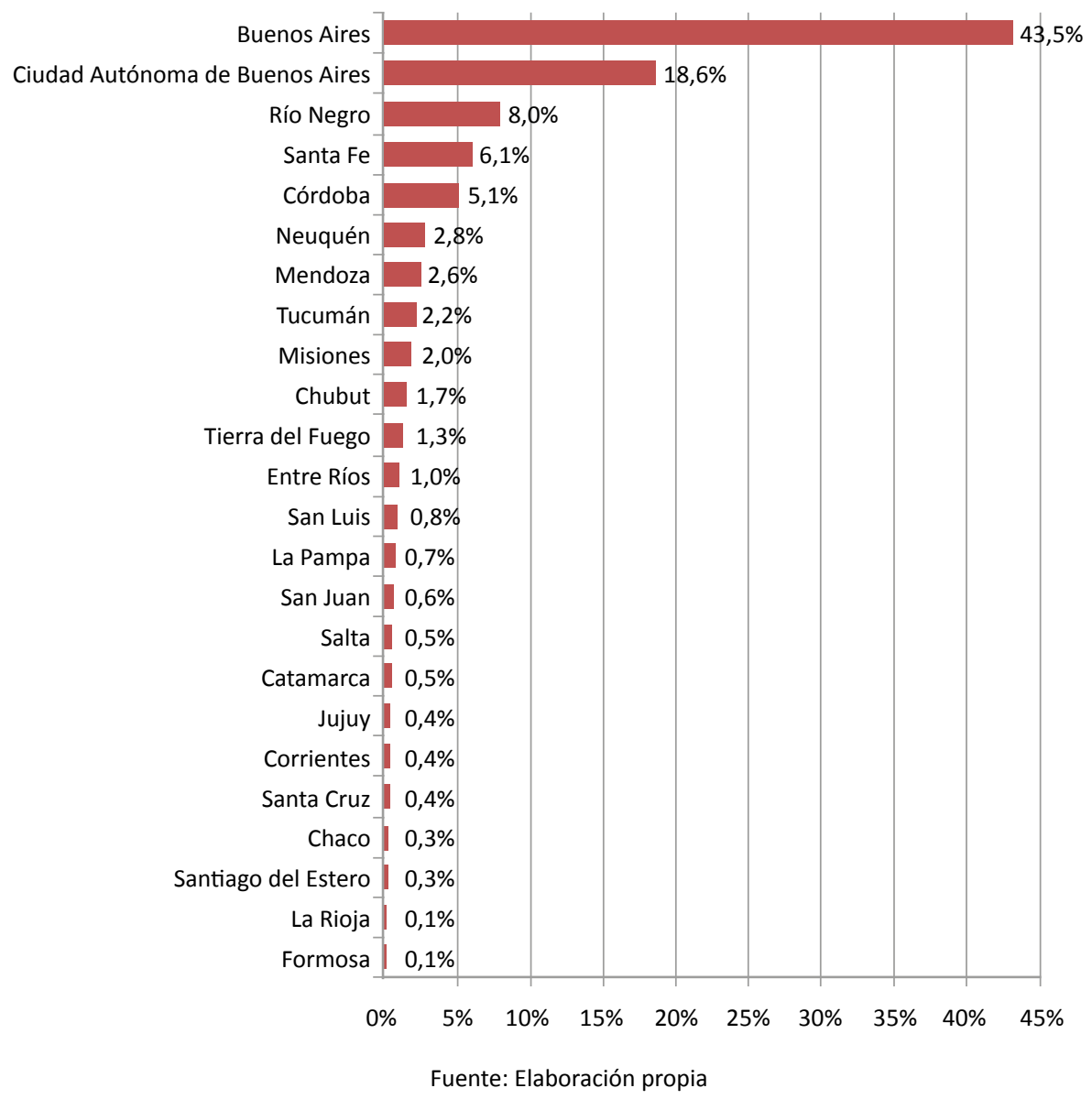

Dado que lo importante en este caso es considerar los efectos directos de las construcciones realizadas o proyectadas en cada provincia, resulta de interés determinar cuánto explican las variaciones en el VAB por cada distrito, para tener una magnitud de las inversiones realizadas.

De acuerdo a lo mencionado Río Negro, es la provincia que más explica su crecimiento económico por los impactos directos de las inversiones. En distritos como Tierra del Fuego y Tucumán, cuatro de cada cinco pesos del crecimiento se originan por las nuevas construcciones, en tanto que en Misiones siete de cada diez pesos. 
Otras provincias en donde prevalecen los efectos de la construcción hotelera y demás obras de infraestructura hotelera son Neuquén, La Pampa y Chubut, con porcentajes que supera el 50\% de la generación de VAB. Estos porcentajes se corresponden con la matriz productiva de cada provincia y particularmente en las patagónicas, donde se localizan gran parte de los principales atractivos turísticos del país.

Estas economías principalmente giran en torno a actividades primarias: agropecuarias, pesca y extractivas ${ }^{2}$, junto con el turismo. Por lo tanto la realización de estas obras son importantes para la generación de valor en actividades que además son de carácter mano de obra intensiva.

\section{Gráfico 2. Participación por provincia del crecimiento del VAB}

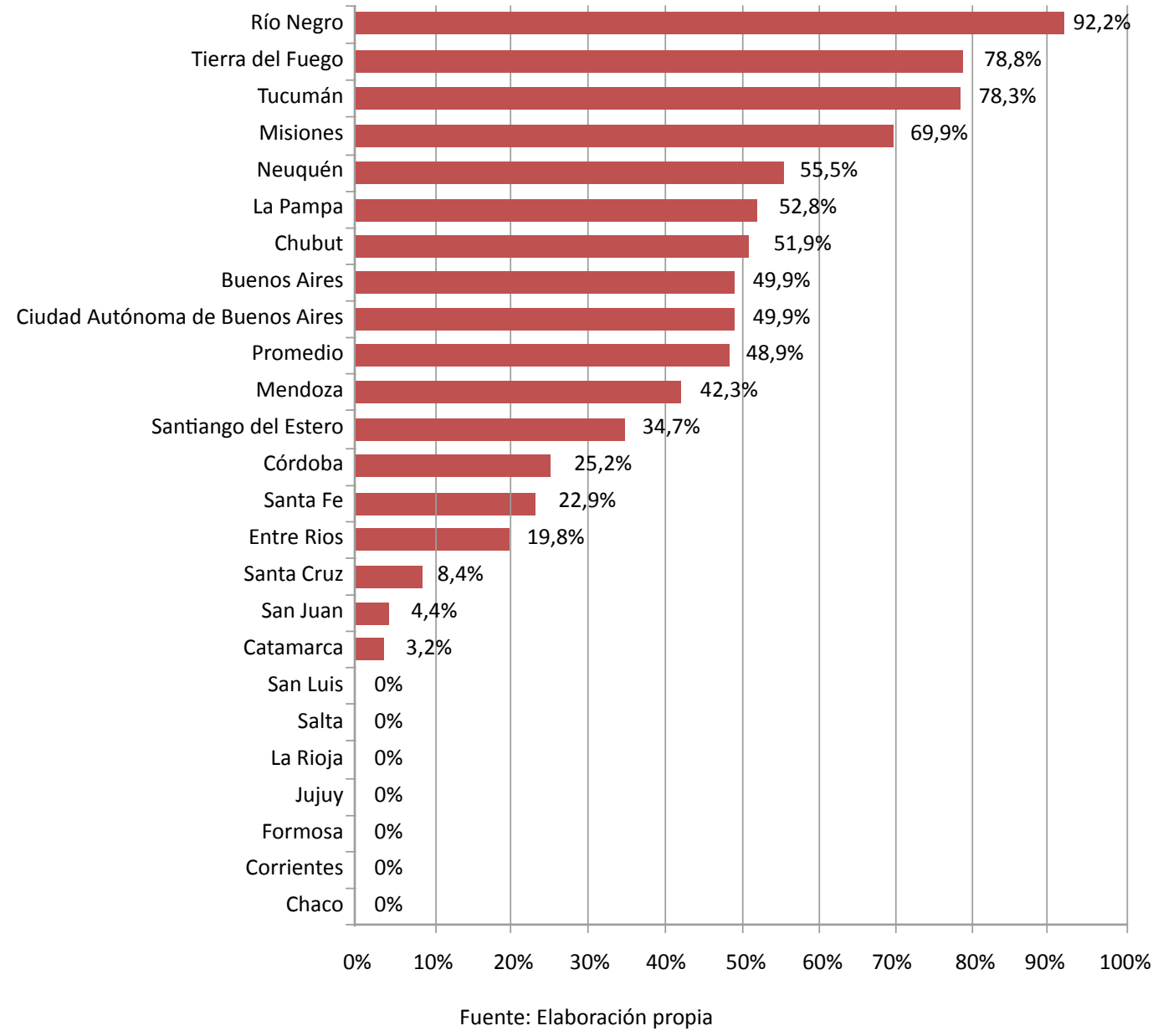

Gracias a la diversidad de paisajes, todas las provincias tienen en mayor o menor medida atractivos turísticos, pero en aquellas que tienen una economía más diversificada, con una importante presencia de actividades industriales, agropecuarias, y de servicios, en términos relativos, la ejecución de las obras parecen no ser tan relevantes; esto se debe a que en esos estados provinciales 
se producen principalmente insumos que después son utilizados en los distritos del país. Por ejemplo el caso de Buenos Aires, Ciudad de Buenos Aires, Córdoba y Santa Fe.

\section{IMPACTO SOBRE LA OCUPACIÓN Y EL INGRESO}

Es quizá uno de los puntos centrales a la hora de determinar la realización de las inversiones, dado que no sólo cabe analizar la factibilidad del proyecto, sino que además, desde el punto de vista técnico la disponibilidad y el acceso a los insumos requeridos en este caso para la construcción.

En este caso la disponibilidad de mano de obra, es un punto central a la hora de llevar adelante construcciones de gran magnitud, dado los requerimientos para llevar adelante los diferentes proyectos. De acuerdo a la información relevada, en algunos proyectos por su envergadura, se requiere la contratación de hasta 500 ocupados del sector de la construcción.

Movilizar esta cantidad de gente puede ser un costo importante para la realización de la obra, con lo cual podría dificultar o al menos, no obtener la tasa de retorno esperada y alargar los plazos de recupero de la inversión. Por lo tanto es necesario en estos proyectos conocer la disponibilidad de trabajadores, especialmente en las localidades del interior del país que se caracterizan por tener poblaciones reducidas y raleadas, especialmente en la Patagonia.

La actividad de la construcción es una importante demandante de mano de obra, por lo tanto cualquier expansión en esta actividad supone contratar a un importante número de trabajadores.

El multiplicador del empleo de la construcción en este caso en infraestructura es de 1,5; esto es, por cada puesto de trabajo creado en este sector en el resto de la economía se crean o,5 ocupados, en actividades que son proveedoras directa o indirectamente de la construcción.

La realización de estas obras supone la demanda de 33.040 puestos de trabajo directos en la construcción por cada año de trabajo. Sobre el total estimado en obras de infraestructura supone alrededor del 5,4\% del total de estos, y considerando el total de la construcción representa cerca del 2,2\%.

Es importante aclarar que este total de puestos de trabajo incluye tanto asalariados, personal contratado, cuentapropistas y socios de las empresas constructoras que realicen alguna actividad, es decir, el total de ocupados, siendo estas las categorías ocupacionales ${ }^{3}$. En el caso

\footnotetext{
${ }^{3}$ Se distinguen tres tipos de categorías ocupacionales: Empleador: las condiciones que organizan los procesos de trabajo en una obra se establecen en un contrato en el que se asigna la denominación de "contratista" a la persona que conduce la unidad productiva que asume el carácter de contratista principal.

Esta cumple la función de empleador con relación a las personas que trabajan a su cargo, y es quien asume la responsabilidad de la dirección de la intervención frente al "comitente" que es el propietario de la obra. El empleador puede ser: • Formal: es el que cumple con alguna de las regulaciones tributarias, contables y laborales que rigen para la industria de la construcción. • Informal: en oposición al anterior, es el que incumple con todas las regulaciones existentes. • Trabajador por cuentapropia: • Formal: es aquel que desarrolla una actividad específica laboral para su propio emprendimiento económico o unidad productiva, cumpliendo con alguna de las normas que regulan sus actividades económicas y las obligaciones previsionales, pudiendo contratar uno o dos ayudantes para desarrollar su tarea. • Informal: es aquel que desarrolla una actividad laboral para su propio emprendimiento económico o unidad productiva, sin cumplir con las normas que regulan las actividades económicas, ni con las obligaciones previsionales. • Asalariado: • Formal: se encuentra trabajando con la protección que establece la legislación laboral vigente. • Informal: se encuentra trabajando sin protección de la legislación laboral. (Ministerio de Trabajo).
} 
de los asalariados se incluyen tanto los formales como informales, que en el caso de este sector se estima que son la mitad de los asalariados.

Además se considera que en promedio dos tercios son asalariados y un tercio lo forman dueños o socios de las empresas constructoras, familiares sin remuneración fija, contratados y cuentapropistas. Dada las características de las empresas aquellas que tienen a cargo grandes obras de infraestructura se reduce la participación de los trabajadores informales. (Ministerio de trabajo)

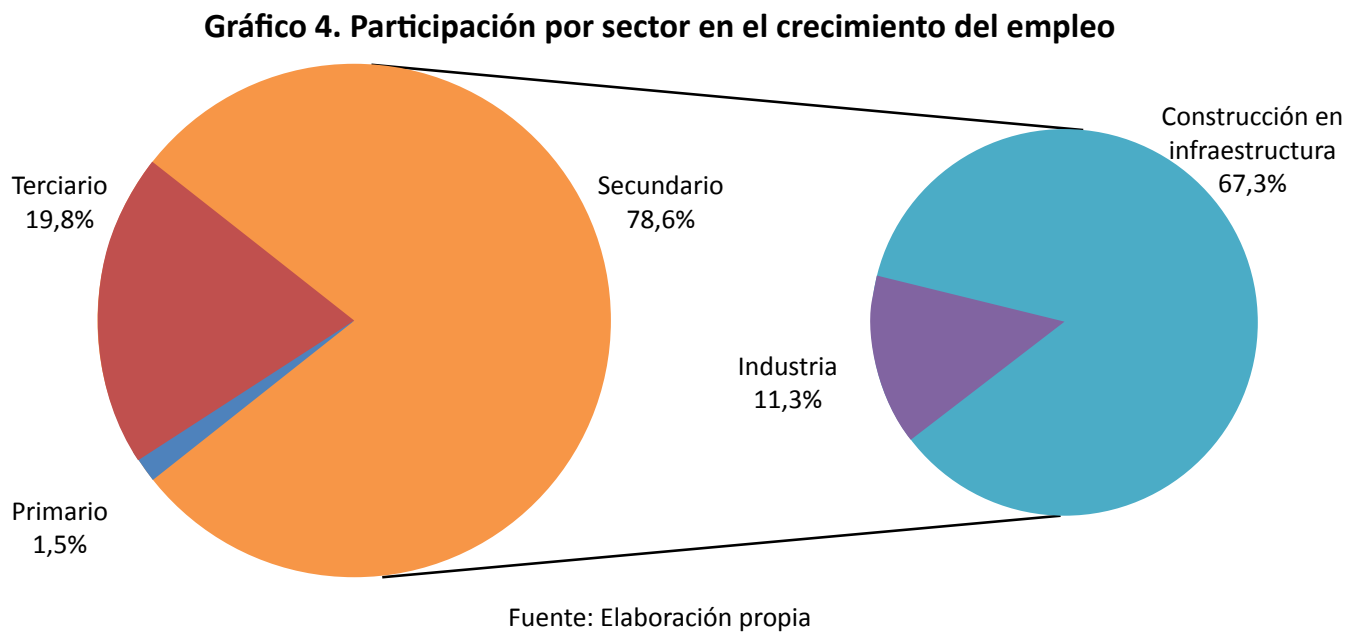

El empleo directo en cada provincia se supone proporcional al valor de las inversiones realizadas en cada una de ellas para su asignación. De este modo se puede estimar la creación de puestos de trabajo por la construcción en infraestructura turística.

Siendo este punto esencial si se desarrollan obras de gran magnitud, donde junto con la cantidad de trabajadores del sector se tiene que tener en cuenta la calificación del personal que lleva a adelante los emprendimientos.

En la provincia de Buenos Aires se requieren alrededor de 14.400 puestos de trabajo por las obras a realizar en forma directa, le sigue la Ciudad de Buenos Aires con 6.155. Entre estos dos distritos se concentran dos tercios de los empleos generados.

Las provincias patagónicas son una importante creadora de trabajos, requiriendo aproximadamente 7.600 empleos, de los cuales casi 5.000 son de la provincia de Río Negro y 1.065 en Neuquén. Se aprecia que en estos distritos, con baja población, la creación de estos empleos que fomenta el desarrollo de estas zonas, evitando la migración. 
Gráfico 5. Estimación de puestos de trabajo creados por provincia

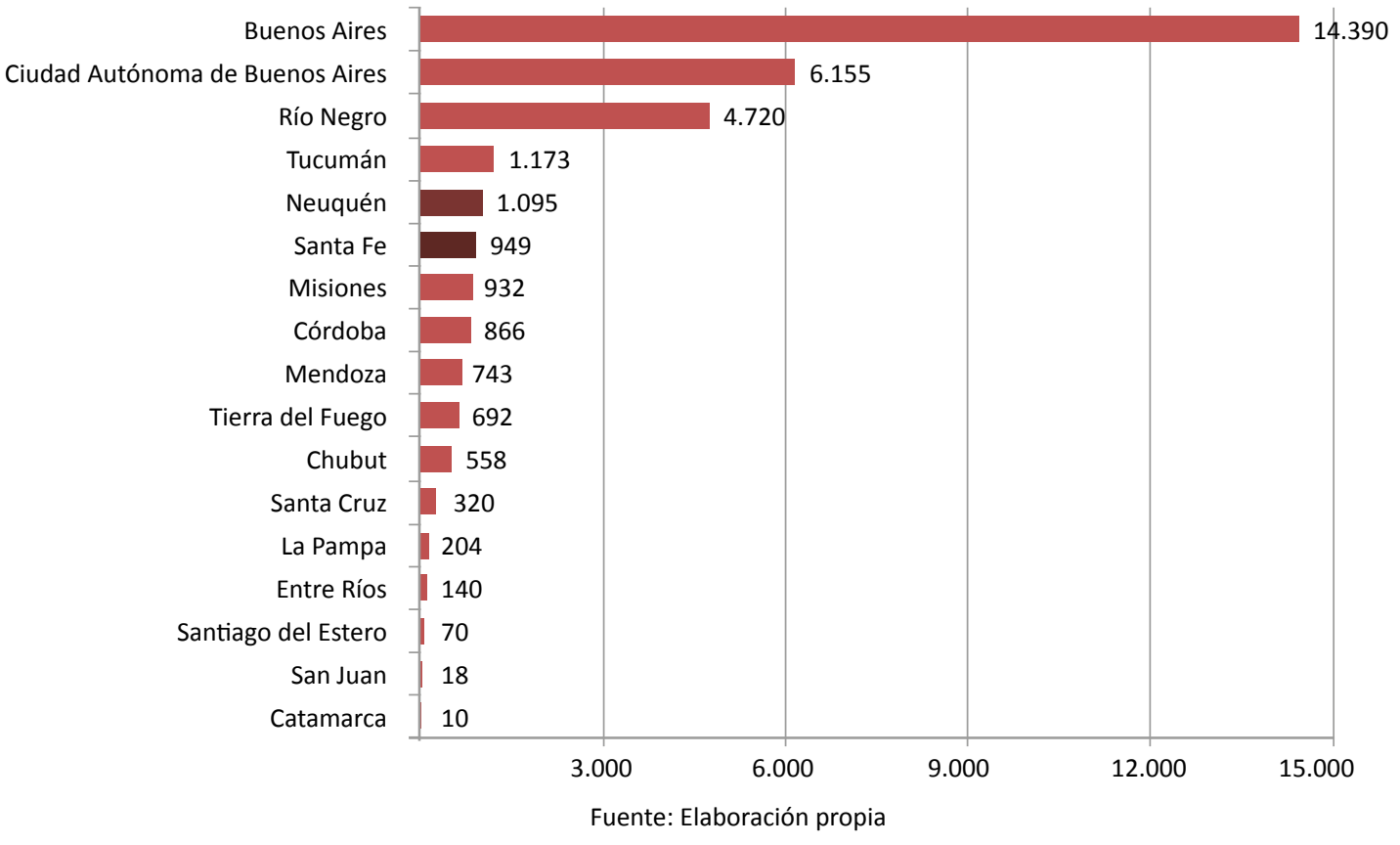

De acuerdo a los datos del Ministerio de Trabajo, Empleo y Seguridad Social de la Argentina, el salario bruto promedio durante el año 2014 en la construcción es de 8.983 entre los asalariados formales.

Este valor medio presenta una gran dispersión de acuerdo a la cantidad de ocupados por empresa constructora. Variando desde los 7.604 pesos en el caso de pequeñas empresas, hasta los 14.270 en aquellas con más de 500 asalariados; esto es casi el doble.

Gráfico 6. Salario promedio bruto en la construcción según cantidad de asalariados por empresa

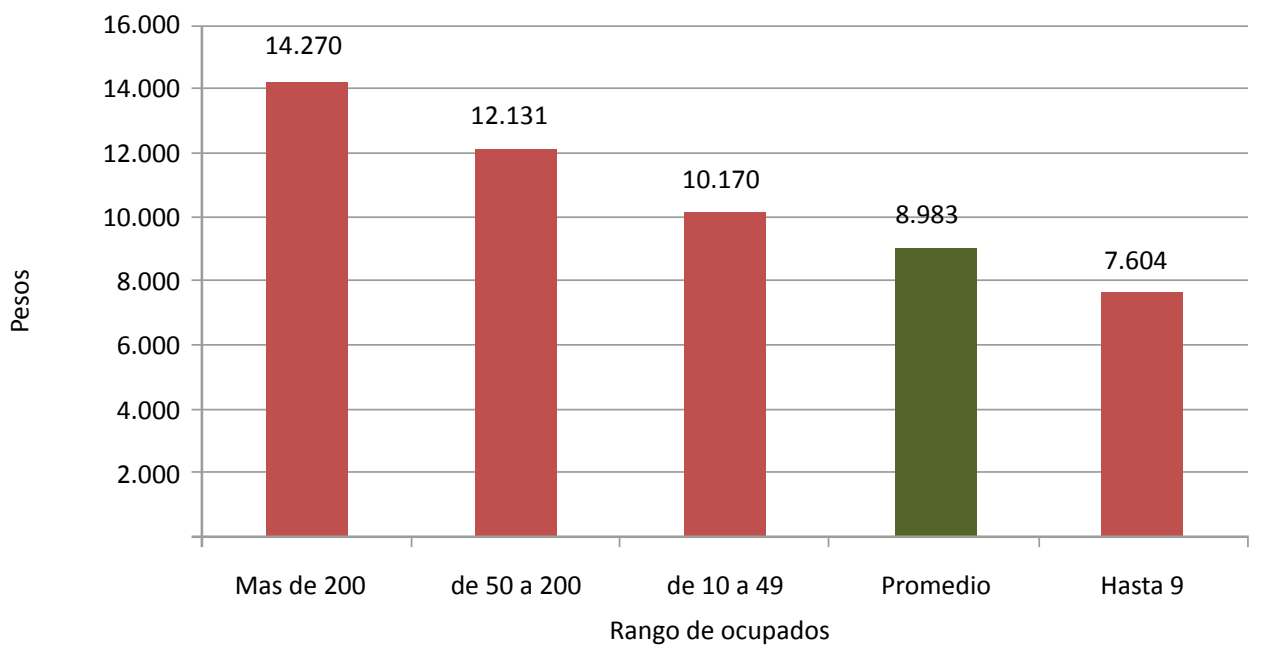

Fuente: Elaboración propia en base a Ministerio de Trabajo, Empleo y Seguridad Social de la República Argentina 


\begin{tabular}{c}
\hline ANA MARÍA GANINO - ADRIÁN GUTIÉRREZ CABELLO \\
$>36$ IMPACTO DE LAS INVERSIONES EN INFRAESTRUCTURA TURÍSTICA. ARGENTINA \\
Págs. $27-43$ \\
\hline
\end{tabular}

La misma situación se observa en los salarios entre las distintas provincias, como los casos de Santa Cruz y Tucumán, que en promedio para el año 2014, son de 22.070 pesos y 5.151 pesos respectivamente; esto es una relación de 4,3 veces superior a favor de la provincia patagónica.

Estas diferencias salariales, existentes entre los distintos distritos obedecen, a factores tanto sociales como económicos, principalmente por la falta de personal en la región sur del país y además la "competencia" existente con los otros sectores que demandan mano de obra como la petrolera, la cual impulsa los salarios de las demás actividades. Es importante mencionar que empresas de la construcción están vinculadas con la explotación de gas y petróleo.

Además las condiciones sociales donde la menor densidad y las largas distancias que se encuentran los centros urbanos de la Patagonia hacen que el costo de vida sean mayores en esa región.

Gráfico 7. Salario promedio bruto en la construcción según provincia. Año 2014

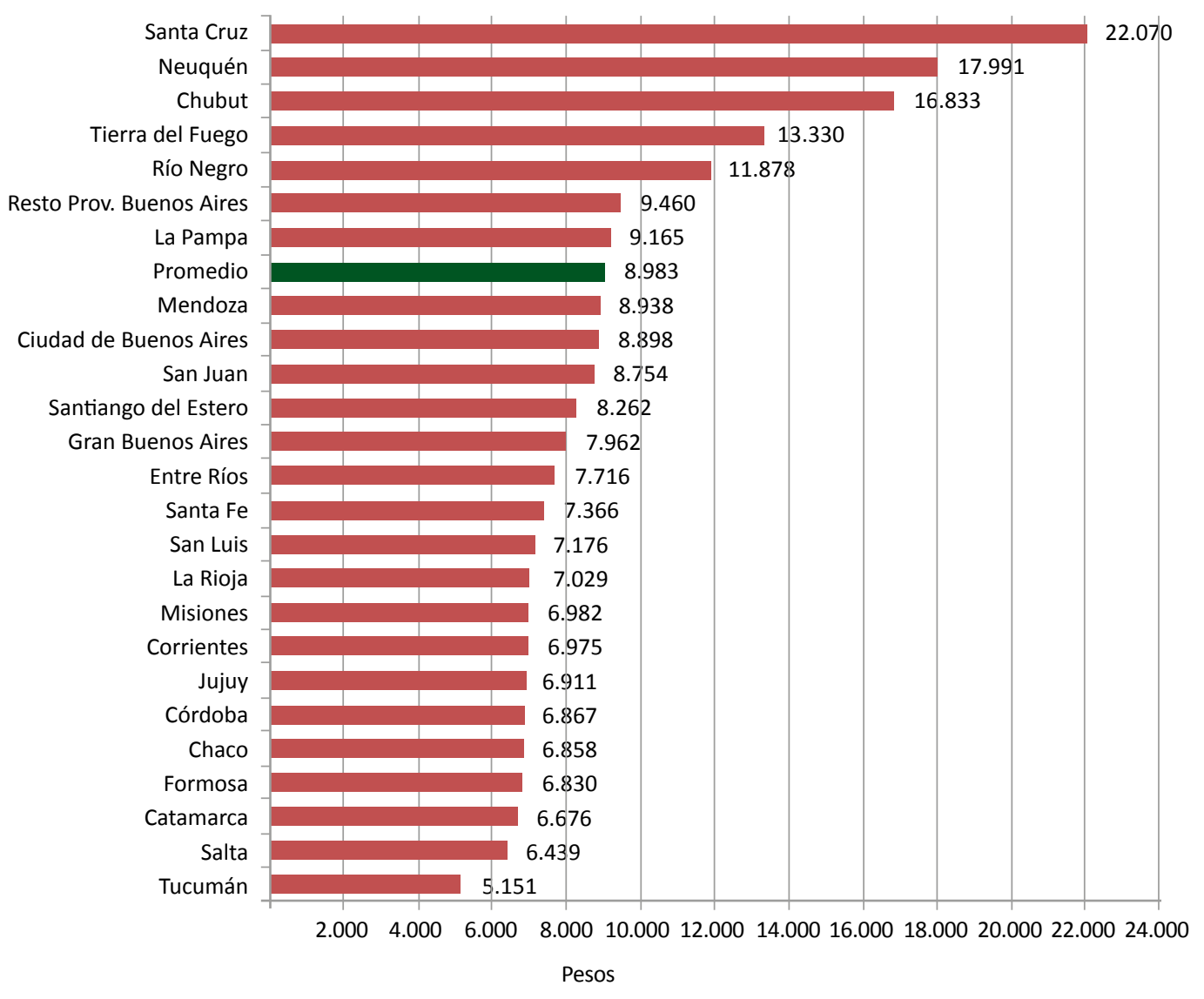

Fuente: Elaboración propia en base a Ministerio de Trabajo, Empleo y Seguridad Social de la República Argentina

Éste es un ítem de consideración a la hora de la realización de un proyecto de inversión dado que los costos de la mano de obra en la construcción varían significativamente entre regiones y provincias. Ello implica la realización de distintos análisis de sensibilidad para es- 
tudiar la factibilidad económica-financiera del proyecto.

Estos salarios son en bruto, a lo cual hay que adicionar las contribuciones patronales y demás gastos (seguros, medicina prepaga, etc.) para determinar el costo laboral por la construcción. Desde el punto de vista social es importante la generación de ingresos en la población local, a partir de los puestos de trabajos creados durante el proceso de la construcción.

Teniendo como referencia que en promedio el $83 \%$ del salario bruto es el que percibe el trabajador y además que todos los puestos de trabajos creados son asalariados formales dada la magnitud de las obras ${ }^{4} \mathrm{o}$ cuentapropistas con ingresos similares a estos.

El total de dinero que percibirían estos trabajadores asciende a 3.421 millones de pesos anuales de acuerdo al salario promedio vigente en cada una de las provincias y considerando la cantidad de puestos de trabajo generados en cada uno de ellos.

Dada la mayor propensión al consumo que tienen estos sectores, cabe esperar que casi la totalidad de este dinero se "gaste" en la propia localidad en la cual se emplazan las obras o en las aledañas, con lo cual se genera un círculo virtuoso en las economías locales. Esto favorece a que se arraiguen las poblaciones, gracias a la demanda de trabajo que tienen en los lugares de residencia.

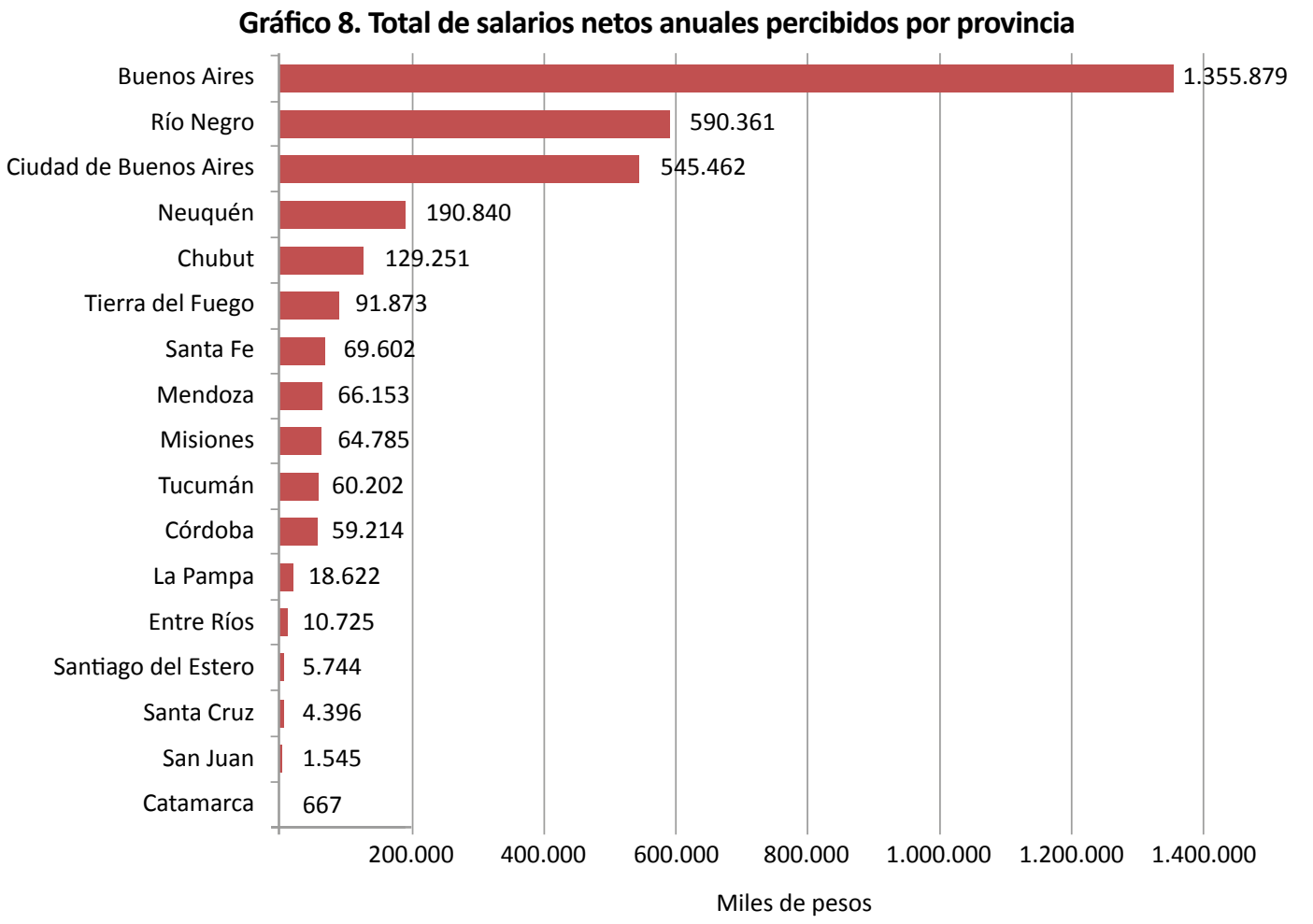

Fuente: Elaboración propia en base a Ministerio de Trabajo, Empleo y Seguridad Social de la República Argentina 


\begin{tabular}{c}
\hline ANA MARÍA GANINO - ADRIÁN GUTIÉRREZ CABELLO \\
$>38$ IMPACTO DE LAS INVERSIONES EN INFRAESTRUCTURA TURÍSTICA. ARGENTINA \\
Págs. 27 - 43 \\
\hline
\end{tabular}

En términos absolutos en la provincia donde se produce un aumento mayor en el ingreso es la provincia de Buenos Aires. La Ciudad de Buenos Aires ocupa el segundo lugar en el destino de las inversiones, pero dado los mayores salarios pagados en Río Negro, este ocupa el segundo puesto en el total de salarios netos percibidos por los trabajadores de la construcción.

Luego siguen en importancia las provincias de Neuquén, Chubut y Tierra del Fuego, que al igual que el otro distrito patagónico, los salarios de esta región son superiores a la media nacional. Las fuertes diferencias en los ingresos, puede acentuar las asimetrías socioeconómicas existentes entre las regiones y provincias, lo cual puede provocar migraciones internas, en búsqueda de mejores condiciones de vida.

\section{IMPACTO DE LA ACTIVIDAD TURÍSTICA POR EFECTO DE LAS INVERSIONES EN EL SECTOR HOTELERO}

El funcionamiento de los hoteles, centros comerciales y demás obras realizadas demandarán personal con mayor o menor grado de calificación, principalmente de las zonas, donde se erigen estos establecimientos. También cabe la alternativa que nuevos trabajadores se radiquen en esas localidades a partir de las mayores oportunidades de trabajo, generadas por la actividad turística.

Dado que no se cuenta con información de cuantos puestos de trabajo se crean en forma directa en los establecimientos hoteleros construidos o a construir, se considera para esta estimación, la presunción de la cantidad mínima de empleados de acuerdo a lo dispuesto por la AFIP. 5

De acuerdo a los datos disponibles, y a fin de simplificar las estimaciones, se considera que en promedio cada hotel contrata 0,8 trabajadores por habitación ${ }^{6}$, sabiendo que la mayoría de los hoteles son de cinco y cuatro estrellas, en estas estimaciones.

\footnotetext{
${ }^{5}$ Resolución 3038 de la AFIP.

${ }^{6}$ Cantidad de trabajadores mensuales por habitación de acuerdo a la resolución antes mencionada, por temporada media para hotel de cinco estrellas.
} 


\section{Gráfico 9. Incremento en el número de habitaciones y de ocupados por las inversiones hoteleras}

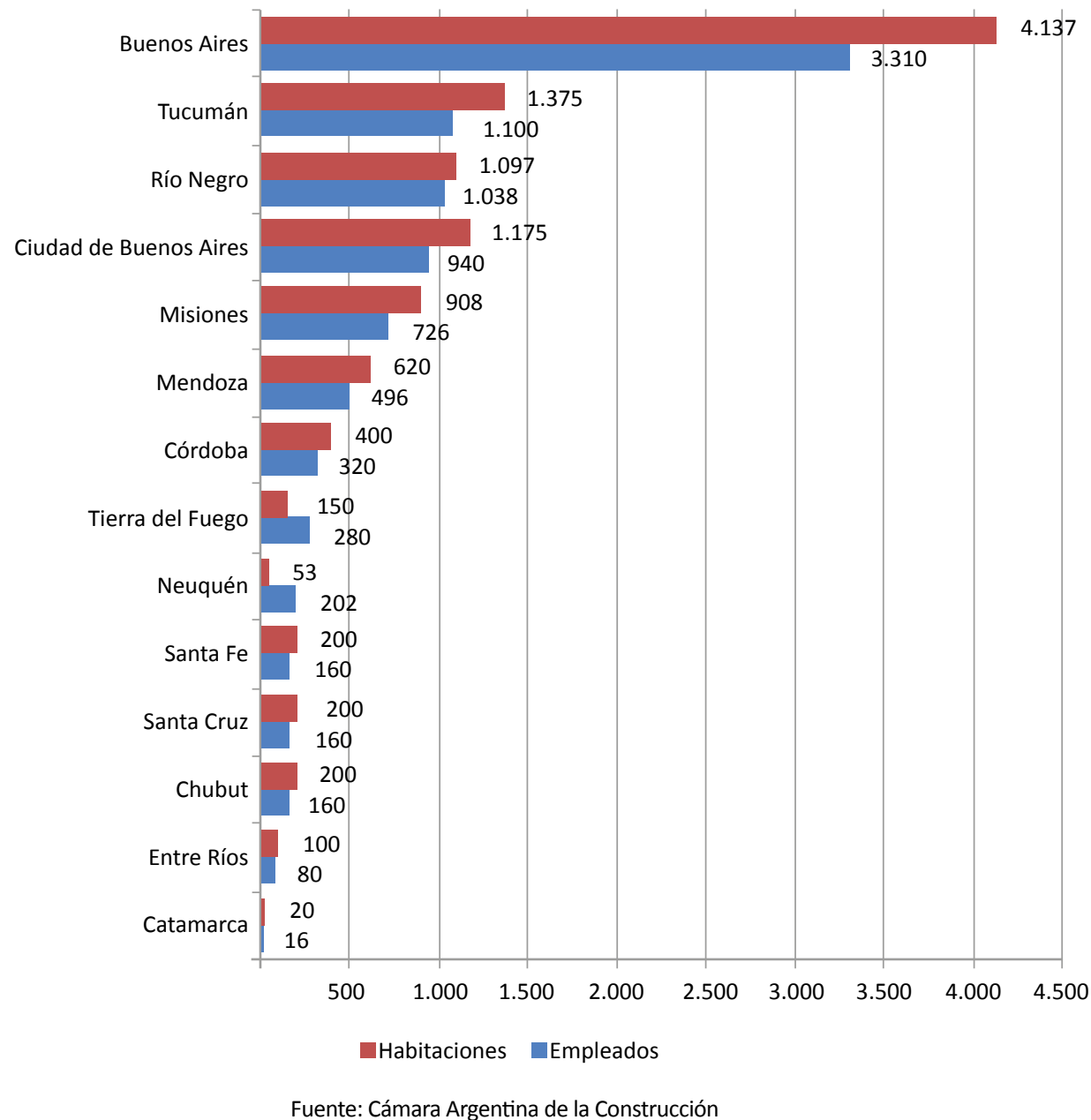

Con la disponibilidad de 11.235 nuevas habitaciones, se requerirá aproximadamente 9.00o empleados en el sector hotelero. Un tercio de estos en la provincia de Buenos Aires, le siguen en importancia Tucumán y Río Negro con el 12,2\% y 11,5\% respectivamente.

Casi un quinto de estos nuevos empleos se crean en las provincias patagónicas, lo cual es un importante impulso en esta región, la más despoblada del país, para arraigar a la población local como así también favorecer la radicación de habitantes en dicho territorio.

En el año 2014 de acuerdo a los datos del Ministerio de Trabajo de la Nación, este sector consta de 269.742 asalariados formales, por lo tanto los 8.988 puestos de trabajos estimados que se crearán implica un crecimiento del 3,3\% del empleo de este rubro.

Si se tiene en cuenta solamente aquellos que trabajan en hotelería representa un incremento del 14\%. Es válido aclarar que no todos los trabajadores de un hotel pueden pertenecer a este sector específico dado que muchos servicios pueden estar tercerizados. 


\section{Gráfico 10. Salario promedio bruto en servicios de hotelería y restaurantes según provincia. Año 2014}

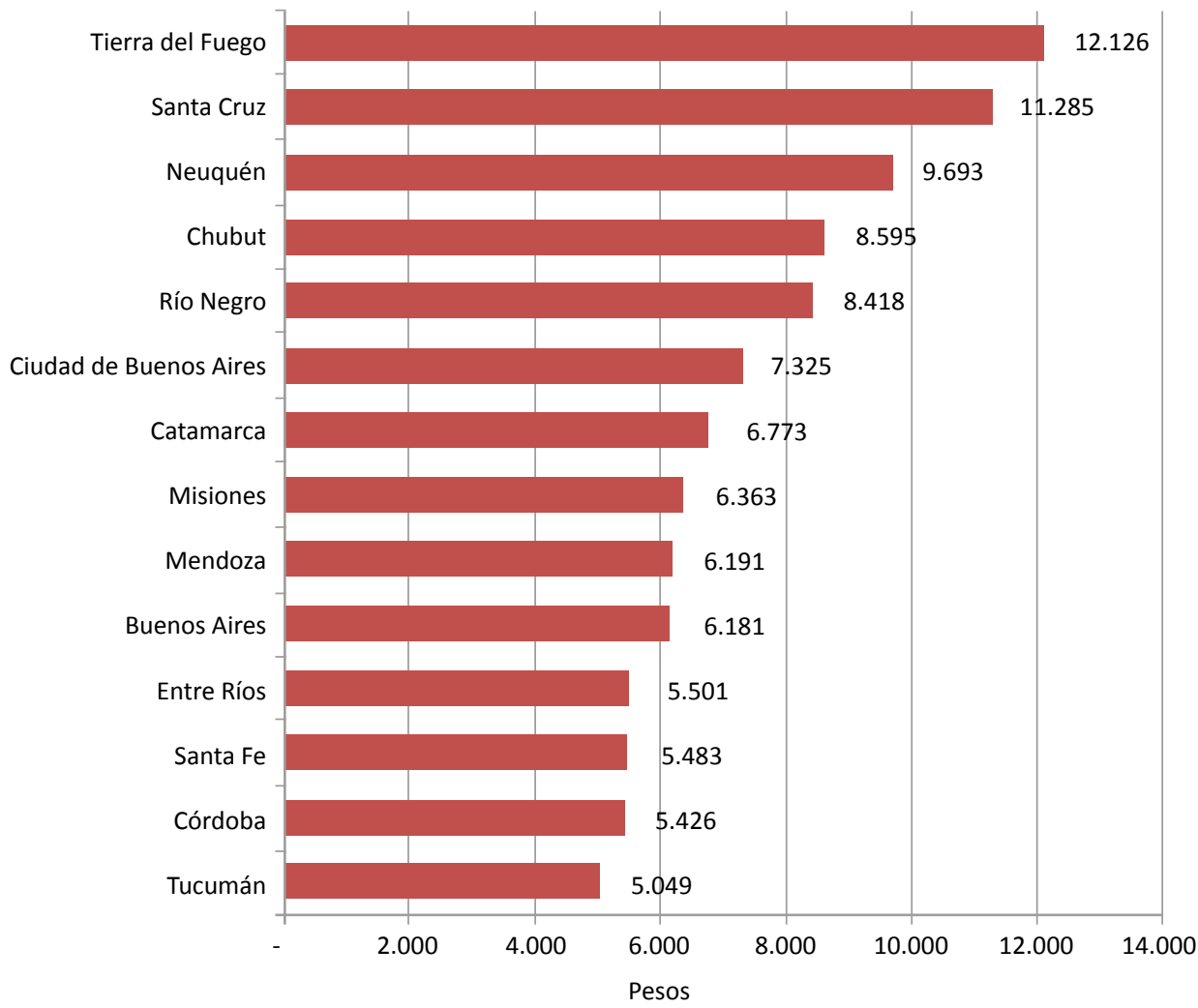

Fuente: Elaboración propia en base a Ministerio de Trabajo, Empleo y Seguridad Social de la República Argentina

En el análisis de los costos, en un proyecto de inversión, los salarios ocupan un papel importante, y esto también es válido para un proyecto hotelero. Las tarifas de hospedaje y demás gastos pueden variar de acuerdo a localización geográfica, lo mismo ocurre con los salarios del personal del sector.

La información a nivel provincial está agregada en servicios de hotelería y restaurantes, tomando como fuente el Ministerio de Trabajo de la Nación. Se observa una importante brecha en el nivel salarial entre los estados provinciales.

La remuneración bruta de los asalariados formales del Tierra del Fuego es de 12.126 pesos, para el año 2014, en tanto que para los de Tucumán asciende a 5.049 pesos; la diferencia entre estos es de 2,4 veces a favor de la provincia patagónica. Este contraste en los salarios es una variable a considerar de relevancia para llevar a cabo el proyecto de inversión.

Las cinco provincias patagónicas, donde se esperan inversiones, tienen los salarios medios más elevados del país en este sector. El promedio simple entre todos los distritos es de 6.876 pesos, por lo tanto implica que los de Tierra del Fuego son casi un $80 \%$ superior y los de la región sur en conjunto el $43 \%$ mayor. ${ }^{7}$

${ }_{7}$ Si el promedio nacional estuviera ponderado por la cantidad de asalariados de cada provincia la brecha sería mayor. 
El total de salarios netos generados por los nuevos puestos de trabajo asciende a 658,4 millones de pesos anuales de acuerdo al salario vigente en el 2014. De este monto casi dos tercios se abonan en la provincia de Buenos Aires, le sigue en importancia, Río Negro, con 94,2 millones.

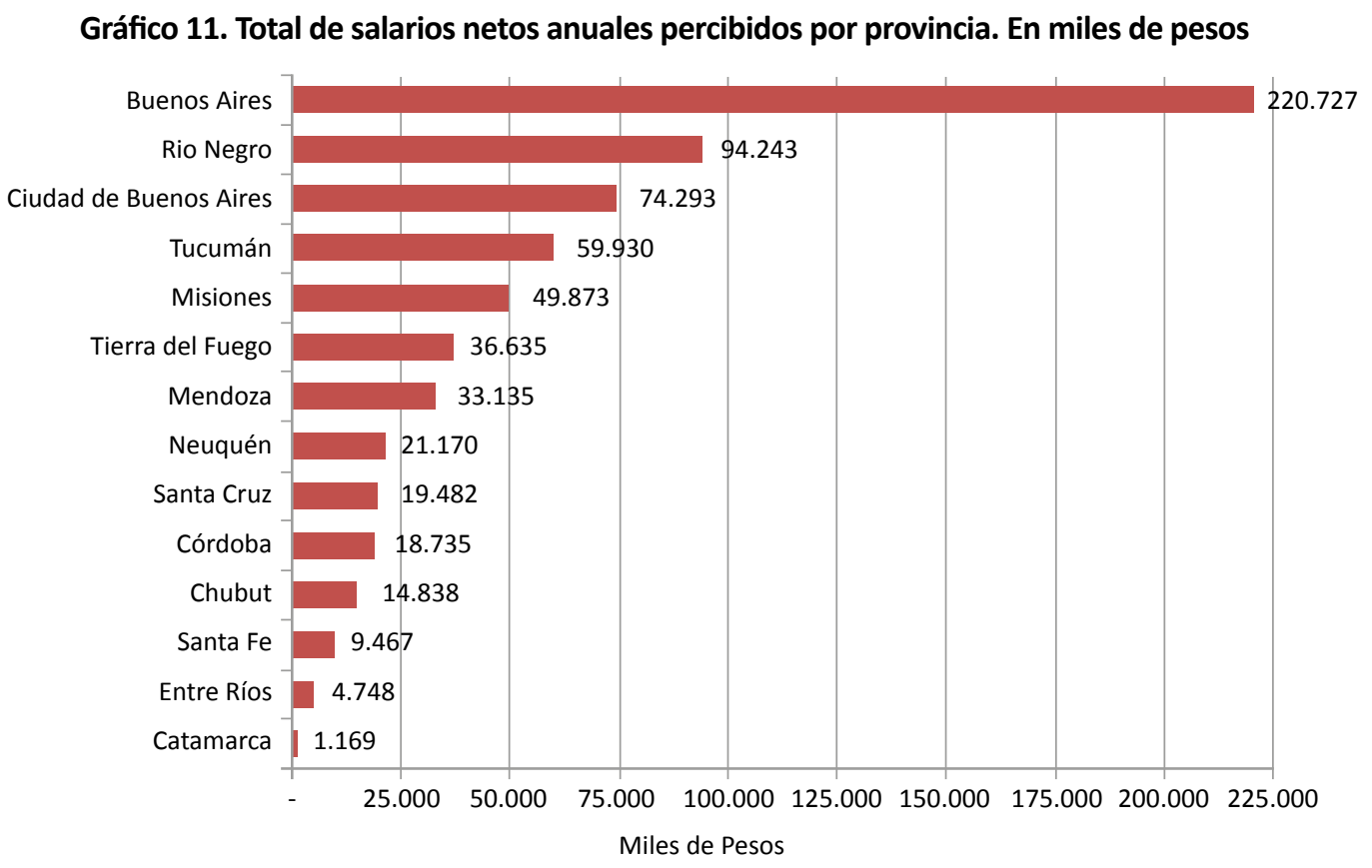

Fuente: Elaboración propia en base a Ministerio de Trabajo, Empleo y Seguridad Social de la República Argentina

Este incremento en la masa de salarios, puede resultar un importante impulso a las economías regionales, especialmente en aquellas provincias con baja densidad de habitantes. De acuerdo a las estimaciones de propensión marginal a consumir y la presión impositiva, cada peso del salario percibido por estos trabajadores, se multiplica por 2,3; con lo cual se produce un potencial foco de desarrollo en las zonas donde se instalen estos hoteles; sin contemplar el gasto que incurren los turistas.

\section{CONCLUSIONES}

La construcción es un factor de impulso a la economía a partir de la gran cantidad de sectores que le proveen insumos y servicios, como así también un gran demandante de mano de obra.

La realización de estas obras de infraestructura y, en particular, en el sector turismo son un importante factor de desarrollo económico y social especialmente en las provincias con menores 
posibilidades de generación de empleo, favoreciendo el arraigo de las poblaciones locales.

Además la demanda de bienes y servicios de la propia región son un factor que alienta a la creación de micro emprendimientos que pueden dar apoyo a la actividad turística en forma sustentable permitiendo la creación de nuevos puestos de trabajo.

Si bien los puestos de trabajo, finalizadas las obras de construcción cesarían, los hoteles, centros comerciales, por ejemplo, demandan nuevos empleos que perduran en el tiempo, por lo tanto los efectos sobre la población son altamente positivos.

Las diferencias de salarios son importantes a nivel provincial, por lo tanto cualquier inversión que se desee realizar debe tenerse en cuenta claramente a la hora de estimar los costos, tanto en la construcción como en la operación de las actividades posteriores.

Es importante resaltar que las inversiones realizadas en este sector a largo plazo, son una fuente importante en la generación de empleo y de valor agregado en la economía, con los consecuentes efectos directos e indirectos, que alientan a una expansión mayor de la actividad productiva.

En síntesis, el presente trabajo pone en relieve la importancia repercusión que tiene la realización de obras de infraestructura con destino a la ampliación de la actividad turística: el fuerte impacto sobre las variables macroeconómicas como arraigarla a sus lugares de origen y los efectos directos e indirectos sobre la necesidad de nuevas inversiones de sectores productivos atraídos por los incrementos derivados de la nueva demanda regional.

\section{REFERENCIAS BIBLIOGRÁFICAS}

AFIP. Resolución General AFIP No 3038/2011.

Cámara Argentina de la Construcción, Anuario 2014. Planes de Inversión. Pulido, A \& Fontela, E. Análisis Input-Output. Módelos, datos y aplicaciones. Ed. Pirámide. Madrid. 1993.

Ministerio de Turismo de la Nación. Anuario Estadístico de Turismo 2014. 


\section{ENLACES CONSULTADOS}

www.economia.gob.ar

www.indec.gob.ar

www.trabajo.gob.ar

\section{CURRICULUM VITAE}

\section{Ana María Ganino}

Lic. en Administración y Gestión Empresarial - Maestría en Economía y Desarrollo del Turismo (Tesis en elaboración). Docente e Investigadora. Especialista en la promoción y desarrollo emprendedor. Docente adjunto por concurso. Investigador Categorizado Nivel V. Consultora y capacitadora. Directora del Proyecto Mapa de inversiones turísticas en Argentina. Universidad Nacional de San Martín - UNSAM.

amganino@unsam.edu.ar

\section{Adrián Gutiérrez Cabello}

Curso de Alta Formación en "Gestión e Innovación en PyMEs. Universidad de Bologna-Italia. Maestría en Generación y Análisis de Información Estadística UNTREF. (Tesis en elaboración). Lic. en Economía UNSAM. Docente adjunto por concurso.. Investigador Categorizado Nivel III. Director de proyectos acreditados. Coordinador del Centro de Economía Regional de la EEyN-UNSAM. Universidad Nacional de San Martín - UNSAM.

acabello@unsam.edu.ar 